\title{
Predicted Properties of the Superheavy Elements. II. Element 111, Eka-Gold ${ }^{1}$
}

\author{
O. L. Keller, Jr., * C. W. Nestor, Jr., Thomas A. Carlson, \\ Oak Ridge National Laboratory, Oak Ridge, Tennessee 37830 \\ and Burkhard Fricke \\ Northwestern University, Evanston, Illinois 60201 and Gesellschaft für Schwerionenforschung, Darmstadt, Germany \\ (Received February 20, 1973)
}

Publication costs assisted by Oak Ridge National Laboratory

\begin{abstract}
The chemical properties of element 111, eka-gold, are predicted through the use of the periodic table, relativistic Hartee-Fock-Slater calculations, and various qualitative theories which have established their usefulness in understanding and correlating properties of molecules. The results indicate that element 111 will be like Au(III) in its chemistry with little or no tendency to show stability in the I or II states. There is a possibility that the 111 - ion, analogous to the auride ion, will be stable.
\end{abstract}

\section{Introduction}

In paper I of this series ${ }^{2 a}$ plans were mentioned for the use of heavy ion accelerators in the search for superheavy elements. These plans have subsequently moved forward in the U. S. and abroad. In the U. S., at the Berkeley Super HILAC, a team of chemists from several U. S. and European laboratories is preparing to study possible superheavy elements. Further predictions are needed now as a guide for such experiments. The tools we have for making chemical predictions are the periodic system of the elements, the relativistic Hartree-Fock-Slater program for calculating energies and radii of electrons in atoms, and various approximate theories which have proven useful over the years in correlating properties of molecules.

If we assume that the periodic table continues to build up with its usual symmetry, element 111 will occur in group $\mathrm{Ib}$ with $\mathrm{Cu}, \mathrm{Ag}$, and $\mathrm{Au}$. The energy eigenvalues presented for $\mathrm{Cu}, \mathrm{Ag}, \mathrm{Au}$, and 111 have been calculated using a relativistic Hartree-Fock-Slater (HFS(rel)) program developed at Oak Ridge ${ }^{2 b}$ and the HFS(rel) code of Fricke and Waber ${ }^{3}$ (a further development of the program of Liberman, Cromer, and Waber $\left.{ }^{4}\right)$. The $6 \mathrm{~d}^{9} 7 \mathrm{~s}^{2}$ groundstate electronic configuration for 111 obtained from both codes was confirmed by a relativistic Hartree-Fock calculation through the courtesy of Dr. Joseph B. Mann. Although the details in the Fricke and Oak Ridge codes differ somewhat, the individual energies given in the tables are, for our purposes, identical. The Oak Ridge code obtains binding energies from the use of Koopmans' theorem and Slater's approximate exchange potential, ${ }^{5}$ whereas Fricke obtains the binding energies from subtraction of total energies and the use of two-thirds times Slater's exchange potential. For well-known reasons neither code can give exact agreement with experiment. Therefore we always compare our calculated values with the available experimental or semiempirical energy values for $\mathrm{Cu}, \mathrm{Ag}$, and Au. As detailed below these comparisons allow us to correct the calculated values for 111 because the physical basis for the errors in the region around element 114 should be the same as for the lighter members of the respective series.

Possible physical changes that are not taken into account in the (HFS(rel)) calculations that conceivably could affect the valence electrons in elements 111 through 120 are (1) the interaction of the electrons with the zeropoint electromagnetic field (vacuum fluctuation); (2) the interaction of the electrons with the polarized negative energy electron sea (vacuum polarization); (3) the electron-electron magnetic interactions; (4) the retardation terms which arise from the finite velocity (speed of light) of the electromagnetic interaction of the electrons as they themselves approach the speed of light; (5) and the error in the Slater exchange term us. exact exchange as found in a relativistic Hartree-Fock calculation. The energy variations due to these causes in the elements around 114 have been found too small to have chemical significance by $\mathrm{Mann}^{2 \mathrm{a}, 6}$ and by Fricke and Waber. ${ }^{7}$ The following assumptions are more serious: (1) the use of Koopmans' theorem by which eigenvalues are used to determine the binding energies, (2) our inability to include electron correlation, and (3) the use of an average energy for an incompletely filled subshell rather than a specific coupling scheme. As shown in the tables, in some instances ionization potentials were obtained from differences in total energies between initial and final states, and in these instances agreement with results from eigenvalues is quite satisfactory. The uncertainties in the latter three assumptions are present for the lighter elements as well as for the heavier elements. We shall therefore assume that the errors in the atomic calculations for element 111 are of the same character as in $\mathrm{Cu}, \mathrm{Ag}$, and $\mathrm{Au}$. The corrections to the energies obtained by comparing the calculated values for $\mathrm{Cu}, \mathrm{Ag}$, and $\mathrm{Au}$ to literature values are therefore assumed to be extrapolatable to 111 . The interpretation of the chemistry of 111 is nonetheless found to be more difficult than for 113 and 114 because the ground electronic state and the electrons of lowest energy are found to be different from those of its congeners. The results we present 
TABLE I

\begin{tabular}{|c|c|c|c|c|c|}
\hline \multirow[b]{2}{*}{ Element } & \multirow[b]{2}{*}{$\begin{array}{l}\text { Configura- } \\
\text { tion }\end{array}$} & \multirow[b]{2}{*}{ Electrons } & \multicolumn{3}{|c|}{ Binding energies, eV } \\
\hline & & & $\begin{array}{c}\text { Oak } \\
\text { Ridge }\end{array}$ & Fricke $^{b}$ & $\begin{array}{c}\text { Semiempir- } \\
\text { ical and ex- } \\
\text { perimental } \\
\text { energies }\end{array}$ \\
\hline \multirow[t]{3}{*}{$\mathrm{Cu}$} & $3 d^{10} 4 s^{1}$ & $3 d_{3 / 2}$ & 10.1 & & 11 \\
\hline & & $3 d_{5 / 2}$ & 9.8 & 10.5 & 10.4 \\
\hline & & $4 s_{1 / 2}$ & 7.1 & 7.7 & 7.73 \\
\hline \multirow[t]{3}{*}{$\mathrm{Ag}$} & $4 d^{10} 5 s^{1}$ & $4 d_{3 / 2}$ & 12.5 & & \\
\hline & & $4 d_{5 / 2}$ & 11.8 & 11.8 & 12.4 \\
\hline & & $5 s_{1 / 2}$ & 6.9 & 7.2 & 7.58 \\
\hline \multirow[t]{3}{*}{$\mathrm{Au}$} & $5 d^{10} 6 s^{1}$ & $5 d_{3 / 2}$ & 12.2 & & 12.5 \\
\hline & & $5 d_{5 / 2}$ & 10.4 & 10.4 & 11.1 \\
\hline & & $6 s_{1 / 2}$ & 8.4 & 8.7 & 9.23 \\
\hline \multirow[t]{3}{*}{111} & $6 d^{9} 7 s^{2}$ & $6 d_{3 / 2}$ & 13.4 & & $(13)^{d}$ \\
\hline & & $6 d_{5 / 2}$ & 10.1 & 10.0 & $(10.7)$ \\
\hline & & $7 s_{1 / 2}$ & 11.7 & 11.9 & $(12.6)$ \\
\hline
\end{tabular}

${ }^{a}$ Based on eigenvalues determined from Oak Ridge code ${ }^{2 a}$ using full Slater exchange $b$ Based on differences in total energies from solutions of wave functions of Fricke $^{3}$ using $2 / 3$ Slater exchange. c Reference 8 . d Suggested extrapolated values for element 111 .

here are therefore of a more general nature than those in paper I.

\section{Energy Levels of Outer Electrons in Group Ib Elements}

In Table I we give the results of the (HFS(rel)) calculations for the energy eigenvalues of the $d_{3 / 2}, d_{5 / 2}$, and $s_{1 / 2}$ electrons of the group Ib elements along with the literature values. The Oak Ridge and Fricke calculations are seen to agree with the literature values ${ }^{8}$ for the binding energies rather well.

As noted previously the ground-state configuration of 111 is $6 d^{9} 7 s^{2}$. This configuration was found to be more stable than the $6 \mathrm{~d}^{10} 7 \mathrm{~s}^{1}$ by 4.2 (Oak Ridge) and by $2.9 \mathrm{eV}$ (Fricke). The first ionization potential of 111 therefore refers to a d electron rather than to an s electron as in the lighter members of the series. Also, as seen in the table, the splitting of the $d_{3 / 2}$ and $d_{5 / 2}$ levels increases through the series to the point that they actually span the $s_{1 / 2}$ level in 111. Whereas the s electron in silver and copper is able to act in some cases more or less as an individual valence electron, some type of hybridization is always involved in gold compounds. In addition to noting the magnitudes of the energies and the relative spacing of the $d$ and $s$ shells, it is also helpful to inspect the computed electron density maps. As seen in Figure 1, at the arrows marking the Slater atomic radii, in copper and silver the $s_{1 / 2}$ density is about equal to the $d_{5 / 2}$, but in $A u$ and 111 it is somewhat less. Also in the tail of the charge distribution at large radii the $s_{1 / 2}$ density is much larger for $\mathrm{Cu}$ and $\mathrm{Ag}$ than the $\mathrm{d}_{5 / 2}$ density whereas in $\mathrm{Au}$ and 111 both these densities are about equal. The probability is therefore low that the $s_{1 / 2}$ electrons in 111 will act individually as valence electrons. That is to say, in 111, as in $\mathrm{Au}$, we can expect hybridization to accompany chemical reaction.

\section{Ionization Potentials and Atomic Radius}

In Table II we give the ionization potentials as obtained by the two HFS(rel) codes as compared to experiment. Agreement between the two codes is again good. Two different approaches are used to obtain the calculated atom-

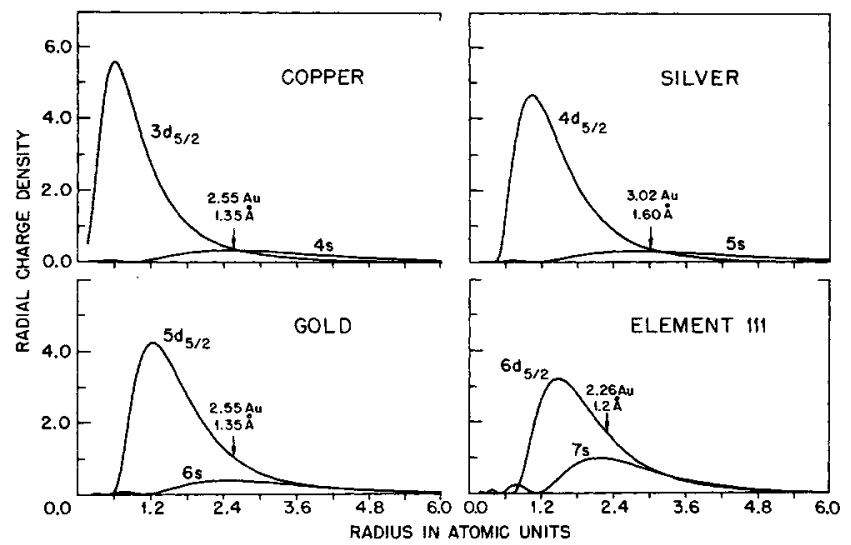

Figure 1. Comparison of $s_{1 / 2}$ and $d_{5 / 2}$ electron densities as calculated by Fricke in group ib elements. The arrows mark the positions of the Slater atomic radii ( $\mathrm{Au}=$ atomic units).

ic radii given in Table II for comparison with Slater's empirical values. ${ }^{9}$ The radial expectation values $\langle r\rangle$ calculated by the Oak Ridge code are listed in ref $2 \mathrm{a}$. From the calculations of Waber and Cromer ${ }^{10 a}$ and of Fricke and Waber, ${ }^{10 \mathrm{~b}}$ we obtained the radius of maximum charge density in the outermost shell. Since, as compared to experiment, the value of $\langle r\rangle$ is consistently too high, and the value of the radius of the charge density maximum is consistently too low, we have no trouble bracketing the "experimental" value of the atomic radius of 111 .

\section{Excited State Energies}

Our subsequent discussions of the chemistry of element 111 will require $s-p, s-d$, and $d-p$ separation energies in the neutral and +1 ion. The results are given in Table III for comparison with available experimental values. The agreement is excellent; so we assume the values for $111^{+}$ and neutral are reliable. Because of their importance to our subsequent discussions, it should be noted that the calculations for the +1 and neutral species both agree that the $d-p$ separation in gold is essentially equal to the s-p separation in 111 .

\section{Relative Stabilities of the Oxidation States of Element 111}

In the absence of complexing agents, and at ordinary temperature, the most stable oxidation state of copper is II and of gold is III. In both cases there is much important chemistry in the I state involving complexes, however. In the case of silver, the most stable and important state by far is the I, with the II having a very high oxidation potential. There is, therefore, no trend as one goes to higher $Z$ in group $\mathrm{Ib}$ that points toward the most stable oxidation state to be expected for element 111, and the predictions must be made on other criteria.

Gold(III) chemistry is nicely explained on the basis of valence bond theory. In gold(III) compounds, which are always four coordinate, the ligands form a square planar arrangement around the gold atom indicating $\mathrm{dsp}^{2}$ hybridization for the gold orbitals. The valence state, as shown in Figure $2 a$, requires the promotion of a $5 d$ electron to a $6 p$ electronic orbital. Au(III) uses all unpaired electrons for bond formation, and also gains an electron pair by forming an anion such as $\mathrm{AuCl}_{4}^{-}$. As shown in Table III, the $\mathrm{d}$ to $\mathrm{p}$ promotion requires $\sim 5.9 \mathrm{eV}$. Therefore, in gold(III) compounds, it is an experimental fact that the bond and 


\begin{tabular}{|c|c|c|c|c|c|c|c|c|}
\hline & \multirow[b]{2}{*}{ Ion } & \multirow[b]{2}{*}{ Configuration } & \multicolumn{3}{|c|}{ lonization energy, eV } & \multirow{2}{*}{$\begin{array}{l}\text { Radial expec- } \\
\text { tation }\langle r\rangle^{d}\end{array}$} & \multirow{2}{*}{$\begin{array}{l}\text { Radius max } \\
\text { density outer } \\
\text { shelle }\end{array}$} & \multirow{2}{*}{$\begin{array}{l}\text { Siater atomic } \\
\text { radius }^{f}\end{array}$} \\
\hline & & & Oak Ridge $^{a}$ & Fricke $^{b}$ & Expt $\left.\right|^{c}$ & & & \\
\hline \multirow[t]{4}{*}{$\mathrm{Cu}$} & neutral & $4 s$ & 7.141 & 7.7 & 7.724 & 1.59 & 1.19 & 1.35 \\
\hline & +1 & $3 d^{10}$ & 19.61 & & 20.29 & 0.50 & & \\
\hline & +2 & $3 d^{9}$ & 37.41 & & 36.83 & 0.47 & & \\
\hline & +3 & $3 d^{8}$ & 58.24 & & & 0.43 & & \\
\hline \multirow[t]{4}{*}{$\mathrm{Ag}$} & neutral & $5 s$ & 6.914 & 7.18 & 7.574 & 1.68 & 1.29 & 1.60 \\
\hline & +1 & $4 d^{10}$ & 20.81 & & 21.48 & 0.69 & & \\
\hline & +2 & $4 d^{9}$ & 35.05 & & 34.82 & 0.67 & & \\
\hline & +3 & $4 d^{8}$ & 50.73 & & & 0.63 & & \\
\hline \multirow[t]{5}{*}{$\mathrm{Au}$} & neutral & $6 s$ & 8.358 & 8.72 & 9.22 & 1.50 & 1.19 & 1.35 \\
\hline & +1 & $5 d^{10}$ & 19.56 & 19.37 & 20.5 & 0.81 & & \\
\hline & +2 & $5 d^{9}$ & 32.30 & 31.79 & & 0.77 & & \\
\hline & +3 & $5 d^{8}$ & 46.20 & & & 0.74 & & \\
\hline & +4 & $5 d^{7}$ & 61.08 & & & 0.71 & & \\
\hline \multirow[t]{5}{*}{111} & neutral & $6 d^{9} 7 s^{2}$ & 10.14 & 9.96 & $(10.7)$ & 1.3 & 1.14 & $(1.2)$ \\
\hline & +1 & $6 d^{8} 7 s^{2}$ & 20.74 & & & 1.3 & 1.12 & \\
\hline & +2 & $6 d^{8} 7 s$ & 30.9 & & & & & \\
\hline & +3 & $6 d^{8}$ & 41.6 & & & & & \\
\hline & +4 & $6 d^{7}$ & 54.5 & & & & & \\
\hline
\end{tabular}

$a-c$ See corresponding footnote to Table $1 .{ }^{d}$ Calculated from solutions for appropriate ions using code from ref $2 a . e$ Calculated from solutions of appropriate ions using code from ref 10 . $f$ Reference 9 .

TABLE III $a$

\begin{tabular}{|c|c|c|c|c|c|c|c|}
\hline & & $\mathrm{Cu}^{+}$ & $\mathrm{Ag}^{+}$ & $\mathrm{Au}^{+}$ & $111^{+}$ & $A u^{0}$ & $111^{0}$ \\
\hline \multirow[t]{4}{*}{$d-s$ separation } & $d^{9} s$ & 2.9 & $4.6(4.6)$ & $1.6(1.6)$ & $-2.1(-2.0)$ & $(0.8)$ & $(2.9)$ \\
\hline & above ground state ${ }^{b}$ & & & & & & \\
\hline & $d^{9} s$ & 2.7 & 4.8 & 1.9 & & & \\
\hline & above ground state $c$ & & & & & & \\
\hline \multirow[t]{4}{*}{$d-p$ separation } & $d^{9} p$ & 8.8 & $9.9(9.8)$ & $7.0(7.7)$ & $4.6\left(d^{10}-d^{9} p\right)$ & $(5.9)$ & (3.3) \\
\hline & above ground state $b$ & & & & $\left(5.9-d^{8} s^{2}-d^{7} s^{2} p\right)$ & & \\
\hline & $d^{9} p$ & 8.2 & 9.9 & 7.8 & & & \\
\hline & above ground state $c$ & & & & & & \\
\hline \multirow[t]{2}{*}{ s-p separation } & $\left(d^{9} s-d^{9} p\right)^{b}$ & 5.9 & $5.3(5.2)$ & $5.4(6.1)$ & $7.1(7.9)$ & $(5.1)$ & $(6.2)$ \\
\hline & $\left(d^{9} s-d^{9} p\right)^{c}$ & 5.5 & 5.1 & 5.9 & & & \\
\hline
\end{tabular}

$a$ Values in eV. ${ }^{b}$ Present work; Fricke results shown in parentheses; Oak Ridge results (as calculated on differences in total energies) shown without parentheses. CJ. D. Dunitz and L. E. Orgel, Advan. Inorg. Radiochem., 2, 1 (1960).

lattice or solvation energies are sufficiently high to furnish this hybridization energy (and also furnish the heat of sublimation of gold) with enough Gibbs' free energy left over to stabilize the complex. Therefore, if the promotion energy to the valence state of 111 (III) is $\sim 6 \mathrm{eV}$, and if it appears that the bond energies of compounds of 111(III) will be about the same or higher than analogous compounds of gold(III), and if it also appears that the heat of sublimation will not be substantially higher, we can then expect that 111 will have a chemistry similar to $\mathrm{Au}(\mathrm{III})$.

(a) $d s p^{2}$ Volence State of Gold (III) [Ground State $5 d^{10} 6 s^{\prime}$ ]

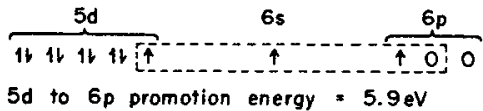

(b) $d s p^{2}$ Valence Stote of Element 111 (III) [Ground State $6 d^{9} 7 s^{2}$ ]

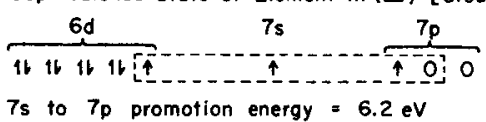

Figure 2. Valence bond pictures for $A u(I I I)$ and 111 (III).
As shown in Figure 2b, the valence state of 111(III) requires the promotion of a $7 \mathrm{~s}$ to a $7 \mathrm{p}$ electron. In Table III, this promotion energy is seen to be $\sim 6.2 \mathrm{eV}$, or about the same as the promotion energy required for $\mathrm{Au}(\mathrm{III})$. The next question concerns the heat of sublimation of 111 . The values of the heats of sublimation of $\mathrm{Cu}, \mathrm{Ag}$, and $\mathrm{Au}$ at $298^{\circ} \mathrm{K}$ are $3.54,2.97$, and $3.67 \mathrm{eV}$, respectively. ${ }^{11}$ There is no trend, and the values are similar. Will the change from $d^{10} s^{1}$ to $d^{9} s^{2}$ ground-state configuration change the heat of sublimation of 111 greatly? A look at the heats of sublimation and ground-state configurations of $\mathrm{Pt}, \mathrm{Au}$, and $\mathrm{Hg}^{11}$ in the following table indicates that the formation of the closed shell $\mathrm{s}^{2}$ configuration will perhaps more than counterbalance the breaking of the $\mathrm{d}^{10}$ closed shell.

$\begin{array}{lccc}\text { Electronic configu- } & \mathrm{Pt} & \mathrm{Au} & \mathrm{Hg} \\ \begin{array}{c}\text { ration } \\ \text { Heat of sublimation } \\ \left(298^{\circ} \mathrm{K}\right), \mathrm{eV}\end{array} & 4 f^{14} 5 \mathrm{~d}^{9} 6 \mathrm{~s}^{1} & 4 f^{14} 5 \mathrm{~d}^{10} 6 \mathrm{~s}^{1} & 4 f^{14} 5 \mathrm{~d}^{10} 6 \mathrm{~s}^{2} \\ & 5.85 & 3.67 & 0.634\end{array}$

The bond energies in 111(III) compounds should be at least as strong as those in $\mathrm{Au}(\mathrm{III})$ compounds because 111 
TABLE IV

\begin{tabular}{ccccc}
\hline & & \multicolumn{3}{c}{ Electron affinities, eV } \\
\cline { 3 - 5 } Element & Configuration & $\begin{array}{c}\text { Oak } \\
\text { Ridge }^{a}\end{array}$ & Fricke $^{b}$ & Expt ${ }^{c}$ \\
$\mathrm{Cu}$ & $3 \mathrm{~d}^{10} 4 \mathrm{~s}^{1}$ & 0.6 & 1.0 & 1.226 \\
$\mathrm{Ag}$ & $4 \mathrm{~d}^{10} 5 \mathrm{~s}^{1}$ & 0.7 & 0.9 & 1.303 \\
$\mathrm{Au}$ & $5 \mathrm{~d}^{10} 6 \mathrm{~s}^{1}$ & 1.4 & 2.1 & 2.3086 \\
111 & $6 \mathrm{~d}^{9} 7 \mathrm{~s}^{2}$ & 1.0 & 1.3 & \\
& & & & \\
& & &
\end{tabular}

is expected to be smaller in radius than Au. Good overlap of its wave functions with those of the ligands should therefore occur. The inner electron repulsion will be larger, but there are indications that this is of minor importance since gold easily achieves $\mathrm{dsp}^{2}$ hybridization whereas $\mathrm{Cu}$ and $\mathrm{Ag}$ do not.

Since the promotion energy and heat of sublimation of 111 should be similar to gold, and the bonding at least as strong, we expect 111 to form strong complexes in the oxidation state of III and be similar to $\mathrm{Au}(\mathrm{III})$ in its chemistry.

There probably will not be a stable oxidation state of II for element 111. Au(II) is exceedingly rare if it exists at all, and $\mathrm{Ag}(\mathrm{II})$ is only difficultly attained. The stability of $\mathrm{Cu}$ (II) relative to $\mathrm{Cu}(\mathrm{I})$ and $\mathrm{Cu}$ (III) is not clearly understood, but it probably involves a delicate balance between ionic and covalent bonding, with the balance tipping toward ionic bonding because of crystal field stabilization. This delicate balance cannot be expected to occur in 111 because of the very high ionization potential of $31 \mathrm{eV}$ vs. 28.0 for copper(II). Even gold(II) with an ionization energy of $29.7 \mathrm{eV}$ does not exist

We expect that $111(\mathrm{I})$ will be very unstable, and that if it exists at all it will be in complexes involving the highly polarizable cyanide ligands which stabilize $\mathrm{Au}(\mathrm{I})$. In Table II we note that 111 has a very high ionization energy of $10.7 \mathrm{eV}$, about $1.5 \mathrm{eV}$ higher than $\mathrm{Au}$. 111(I) can therefore be expected to be noble indeed. Furthermore, $\mathrm{Cu}(\mathrm{I})$ and $\mathrm{Ag}(\mathrm{I})$ are stabilized by a great change in radius that occurs when a single electron is ionized. As shown in column 7 of Table II, a decrease of about $1 \AA$ occurs in these two cases. The decrease in radius in going to the +1 ion from the neutral species results in much larger lattice energies in crystals and solvation energies in solution than would otherwise be the case. For gold the corresponding decrease is seen to be somewhat less and for 111 no decrease occurs. Gold forms a quasistable I state with covalent properties. For the case of 111 , the high ionization potential and small change in radius can be expected to preclude the formation of 111 (I) except, perhaps, with cyanide.

Another intriguing possibility for a stable oxidation state of 111 is the -1 ion analogous to the auride ${ }^{12}$ ion found in CsAu and RbAu. Cesium auride is a semiconduc- tor whose bonding appears to be highly ionic. The crystals have the $\mathrm{CsCl}$ structure. Whether 111 will act in a similar way to $\mathrm{Au}$ depends on its electron affinity. The electron affinity of gold ${ }^{13}$ is about $2.3 \mathrm{eV}$. The electron affinities of $\mathrm{Cu}$ and $\mathrm{Ag}$, which have not been found to form compounds containing themselves as -1 ions, are about 1.2 and $1.3 \mathrm{eV}$, respectively.

In order to evaluate whether 111 will act like gold or like copper and silver in this instance, we need to be able to compute the electron affinity. This is a difficult problem, even in the lighter elements, because electron-electron correlation assumes such a major role. ${ }^{14,15}$ Even though our codes could not be reasonably expected to give accurate results, we nevertheless carried out the calculations as a matter of interest for purposes of comparison. The results, given in Table IV, are seen to be in the correct order with $\mathrm{Cu}$ and $\mathrm{Ag}$ about equal and $\mathrm{Au}$ somewhat higher. According to these calculations element 111 is seen to lie between $\mathrm{Au}$, which forms a negative ion in chemical compounds, and $\mathrm{Cu}$ and $\mathrm{Ag}$ which do not. We would therefore suggest strongly that experimentalists be aware of the possible stability of the $111-$ ion, and its possible chemical importance.

Acknowledgment. We wish to express our appreciation to Professor Glenn T. Seaborg for pointing out to us the importance of chemical predictions in the superheavy elements region. We also wish to thank him for numerous stimulating discussions during the course of this work.

\section{References and Notes}

(1) Research sponsored by the U.S. Atomic Energy Commission under contract with the Union Carbide Corporation.

(2) (a) O. L. Keller, Jr., J. L. Burnett, T. A. Carlson, and C. W. Nestor, Jr., J. Phys. Chem., 74, 1127 (1970); (b) C. C. Lu, T. A. Carlson, F. B. Malik, T. C. Tucker, and C. W. Nestor, Jr., Atomic Data, 3 (1971).

(3) B. Fricke, W. Greiner, and J. T. Waber, Theor. Chim. Acta, 21, 235 (1971); B. Fricke and W. Greiner, Phys. Lett. B, 30, 317 (1969).

(4) D. Liberman, D. T. Cromer, and J. T. Waber, Comp. Phys. Commun., 2, 107 (1971).

(5) J. C. Slater, Phys. Rev., 81, 385 (1951); see also W. Kohn and L. J. Sham, Phys. Rev., 140, A 1113 (1965); R. D. Cowan, A. C. Larson, D. Liberman, J. B. Mann, and J. T. Waber, Phys. Rey., 144, 5 (1966).

(6) J. B. Mann, J. Chem. Phys., 51, 841 (1969); J. B. Mann, Robert A. Welch Foundation Conference. The Transuranium Elements, Houston, Tex.. Nov 1969.

(7) B. Fricke and J. T. Waber, J. Chem. Phys., 57, 371 (1972).

(8) W. Lotz, J. Opt. Soc. Amer., 60, 2061 (1970); M. O. Krause, "Electron Energy Levels in Free Atoms from $Z=1$ to $Z=60$," ORNLTM-2943, April 1970; C. E. Moore, "Atomic Energy Levels," National Bureau of Standards, 1958.

(9) J. C. Slater, "Quantum Theory of Molecules and Solids," McGrawHill, Vol. 2, New York, N. Y., 1965, p 103.

(10) (a) J. T. Waber and D. T. Cromer, J. Chem. Phys., 42, 4116 (1965); (b) B. Fricke and J. T. Waber, ibid., 56, 3246 (1972).

(1.1) D. R. Stull and G. C. Sinke, Advan. Chem. Ser., No. 18, 1 (1956)

(12) (a) A. Sommer, Nature (London), 152, 215 (1943); (b) W. E Spicer, A. H. Sommer, and J. G. White. Phys. Rev., 115, 57 (1959).

(13) H. Hotop, R. A. Bennett, and W. C. Lineberger, Proceedings of the Twenty-Fifth Annual Gaseous Electronics Conference, 17-20 Oct 1972. The University of Western Ontario, London, Canada.

(14) (a) E. Clementi and A. D. McLean, Phys. Rev., 133, A419 (1964); (b) E. Clementi, A. D. McLean, D. L. Raimondi, and M. Yoshimine, Phys. Rev., 133, A1274 (1964).

(15) B. L. Moiseiwitsch, Advan. At. Mol. Phys., 1, 61 (1965). 